\title{
DESIGN AND IMPLEMENTATION OF A RADIO CONTROLLED LED LIGHTING SYSTEM \\ ${ }^{1}$ A. Bálint, ${ }^{2}$ J. Sárosi \\ ${ }^{1}$ Technical Institute, Faculty of Engineering, University of Szeged, Moszkvai krt. 9,6725, Szeged, Hungary, e-mail: balint-a@mk.u-szeged.hu \\ ${ }^{2}$ Technical Institute, Faculty of Engineering, University of Szeged, Moszkvai krt. 9,6725, Szeged, Hungary, e-mail: sarosi@mk.u-szeged.hu
}

\begin{abstract}
Technological advances allow us to control LED (Light Emitting Diode) lighting at home, in the office it can even allow us to change the intensity of the light and this is just the beginning of what the future holds for us. In this study an LED internal lighting fixture of our own design will be presented focusing on each step in its design. Firstly, the features, types and history of LEDs will be presented, next the products available in market that are required for the completion of the device. Finally, the design and implementation as well as the radio frequency controlled LED indoor lighting fixture will be shown.
\end{abstract}

Keywords: LED, lighting device, microcontroller, radio frequency

\section{INTRODUCTION}

LED technology has gone through a rather fast development as LED efficiency and light intensity is increasing so does it gain a greater foothold in lighting technology and everyday use thus we cannot ignore it as an everyday light source. The secret to the LEDs worldwide conquest and long term success lies in its small size, virtually limitless lifespan and high reliability as an added safety feature the ultra-low voltage required by LEDs make them unique in applications that require electric shock protection [1].

A physical classification of LEDs places them as electroluminescent radiating layered diode in which a P$\mathrm{N}$ layer can be found. A method of comparison can be the luminous flux emitted per unit of power, this also helps divide the application areas [2], [3].

LED ribbons (also known as LED cables) are used to replace traditional light cables as they are more energy efficient. These light sources are no longer manufactured in circular plastic pipes, but on thin flexible PCBs (Printed Circuit Boards). One of the advantages of the flat design is that the light sources always face the same direction making for an even light distribution unlike the pipe design where the direction of the micro light source is mostly random. Another advantage of this design is that the light radiates in a $120^{\circ}$ cone, the advantage of this is that all the light goes to producing the light effect as well as not shining through once placed in a polystyrol frame. The LED chip or SMD (Surface Mounted Device) LED are placed lying on the surface unlike DIP (Dual Inline Package) LEDs which stand on their legs. This feature gives them better heat dissipation as well as greater mechanical stability.

From the lighting experience point of view the COB (Chip On Board) LEDs advantage over other LED-s is that the light is generated over a large area enabling fixtures that provide ample lighting but do not blind or dazzle. A MCOB (Multi Chip On Board) LED does not provide light in one great area but rather smaller ones in "cups". The result of continuous improvement to the LED technology is the OLED (Organic LED) and its sub types the PLED (Polymer LED), PHOLED (Phosphorescent OLED), TOLED (Transparent OLED) other types are still in development [4], [5], [6], [7].

Radio frequency control can be achieved with a simple analog circuit which via frequency or amplitude modulation can provide simplex data transmission for us. In the majority of cases the source and destination for the data to be sent is a microcontroller, and the data are transmitted in digital format. Given this one of the best solutions is to use a transceiver module such as the NRF24L01. A great advantage of this type of modules over traditional transmitters and receivers is that it can achieve full-duplex data transmission, as well as using a protocol where data packets can be addressed, errors can be detected, and 
in case of errors resend the failed packets automatically. Tab. 1 shows some of the currently used radio frequency modules that are commercially available.

Table 1. Comparison of radio frequency modules

\begin{tabular}{|l|c|c|c|c|}
\hline & & & \\
& & & & \\
& & & & \\
\hline Name & NRF24L01 & Bluetooth & ZigBee & Z-Wave \\
\hline Frequency & $2.4 \mathrm{GHz}$ & $2.4 \mathrm{GHz}$ & $2.4 \mathrm{GHz}$ & $868 \mathrm{MHz}$ \\
\hline Max. packet size & $33 \mathrm{byte}$ & $1024 \mathrm{byte}$ & $127 \mathrm{byte}$ & $21 \mathrm{byte}$ \\
\hline Transfer speed & $2 \mathrm{Mb} / \mathrm{s}$ & $237 \mathrm{~Kb} / \mathrm{s}$ & $250 \mathrm{~Kb} / \mathrm{s}$ & $100 \mathrm{~Kb} / \mathrm{s}$ \\
\hline Applicable distance & $50 \mathrm{~m}$ & $10 \mathrm{~m}$ & $150 \mathrm{~m}$ & $100 \mathrm{~m}$ \\
\hline Price & $0.85 €$ & $3.18 €$ & $25.44 €$ & $50.89 €$ \\
\hline
\end{tabular}

Bluetooth is a standard with automatic packet handling, which provides a two way data channel in which each master device can connect up to a maximum of 7 other devices, thus one controller unit can control several units. Furthermore the Bluetooth module is rather inexpensive and unlike the NRF24L01 nearly all mobile phones are equipped with Bluetooth thus no further converters are needed.

The ZigBee is developed by the ZigBee Alliance and is built upon the IEEE 802.15 standard. It works in the $2.4 \mathrm{GHz}$ frequency range, uses 16 channels and has a maximum transfer speed of $250 \mathrm{~Kb} / \mathrm{s}$. Thanks to the AES-128 it supports encryption with a passphrase providing a secure communication. It can also work in energy saving mode and its range varies between 50-500 $\mathrm{m}$ depending on the conditions.

The Z-wave was developed by the Danish Zensys company and is currently being developed by the American Sigma Design. This module builds a self-managing and self-repairing network, using routing tables and neighbour tables to build these routes. Depending on the region they work in different frequencies: $868.42 \mathrm{MHz}$ (EU), $908.42 \mathrm{MHz}$ (USA), $921.42 \mathrm{MHz}$ (Australia) and has a maximum transmission speed of $100 \mathrm{~Kb} / \mathrm{s}$.

The NRF24L01 is produced by Nordic Semiconductors, works in the $2.4 \mathrm{GHz}$ range, has a maximum transfer rate of $2 \mathrm{Mb} / \mathrm{s}$ with low power consumption, it also has a trade-off between distance and transfer speed. Configuration and operation is possible through the high speed SPI bus. Drawbacks: the module can only have a maximum of 5 open communications if we desire larger networks additional programming is required, furthermore the module has no encryption by default and has to be programmed if need be. From these four modules the most suitable has been chosen based on the parameters given which is the nRF20L01. The decision was made based on the total price of the electronic controller, the ability to connect more modules in a network, low power consumption, and the range should be the longest.

\section{DESIGNING A LED LIGHTING SYSTEM}

The task presented to us was the replacement of the chandelier which had spotlights and provided the illumination for main room in an apartment. The replacement should be more efficient, have more intense lighting, and consume less power. The spotlights consumed over $200 \mathrm{~W}$ of power and provided inadequate illumination. Thus the need arouse to create a ceiling lighting fixture which adequately illuminated the room, consumed less power and had adjustable light intensity. These criteria could only be accomplished by LED lighting. The unique design provided a way to adjust the light intensity allowing for increased energy efficiency and mood lighting [8]. 
The first step was the design of the frame of the lighting fixture. This was done using the Autodesk Inventor program (Fig. 1). The pieces were cut from wooden boards, in the design the following had to be taken into consideration: the individual board segments should be small as possible, the individual segments should look the same, it should be easy to cut out, and the angles should be $90^{\circ}$ or $45^{\circ}$. During assembly wooden screws and glue were used to increase the strength of the frame. Finally, the frame had been painted to the desired colour.

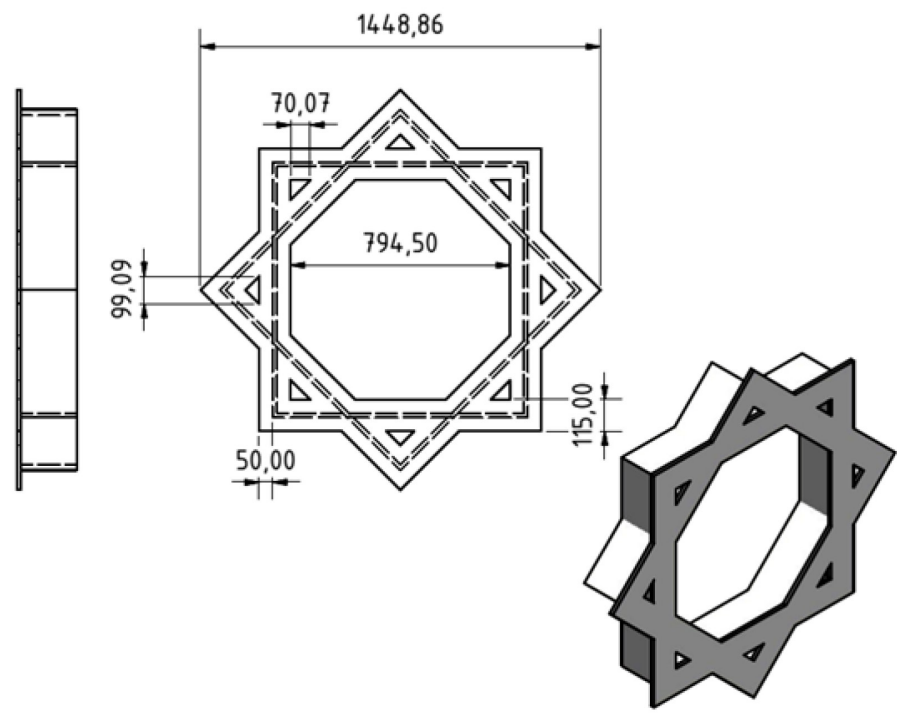

Figure 1. Scheme of the chandelier frame

The next step was the selection of the LED strip. A 12 V 5630 warm white LED strip was chosen which contains 60 LED-s per meter. The 5630 specifies the LED length to be $5.6 \mathrm{~mm}$ and width to be $3 \mathrm{~mm}$. The total length required was $8 \mathrm{~m}$ which requires $8 \mathrm{~A}$ of current. To provide the required current an open factory standard $120 \mathrm{~W}$ power supply was chosen, this drives the control electronics as well.

Fig. 2 depicts a block diagram of the control circuit and its subsystems. The block diagram contains the main subsystems: power supply, AVR microcontroller, and NRF24L01 radio frequency module. From the block diagram it can be seen that three levels of voltage are required. The first is the power supply output of $12 \mathrm{~V} \mathrm{DC}$ converted from $230 \mathrm{~V} \mathrm{AC}$, this powers the LED strips. The second is $5 \mathrm{~V}$ for the microcontroller and finally the $3.3 \mathrm{~V}$ required for the radio module. Since the LEDs and the microcontroller do not use the same voltage converters were used.

The controller selected for this task was the ATmega328 microcontroller from Atmel. This 8 bit microcontroller is rated for 20 MIPS meaning it performs 20 million operations per second if a $20 \mathrm{MHz}$ clock signal is applied [9], [10]. The controller also requires a quartz crystal oscillator to set the clock speed. 


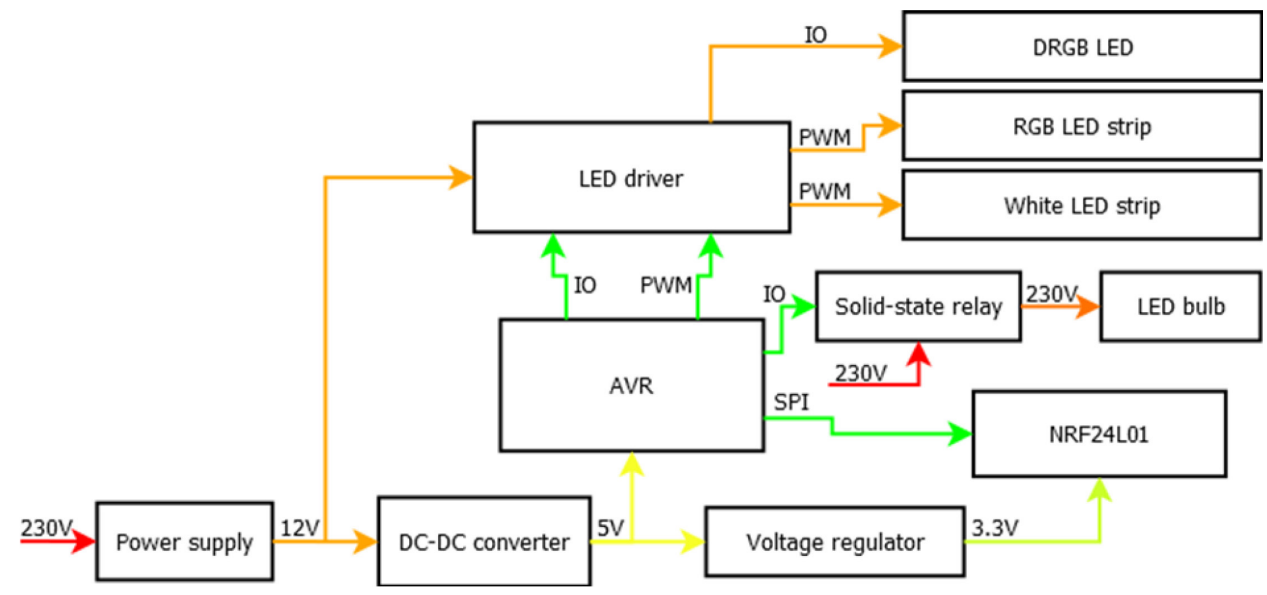

Figure 2. Block diagram of the control circuit

The circuit diagram (Fig. 3) was created using the EAGLE (Easily Applicable Graphical Layout Editor) program, which has the benefit of creating a printed circuit board layout from the circuit diagram (Fig. 4). The printed circuit boards were created using ironing and etching techniques. After the checking of the placement and the soldering the controller program was written.

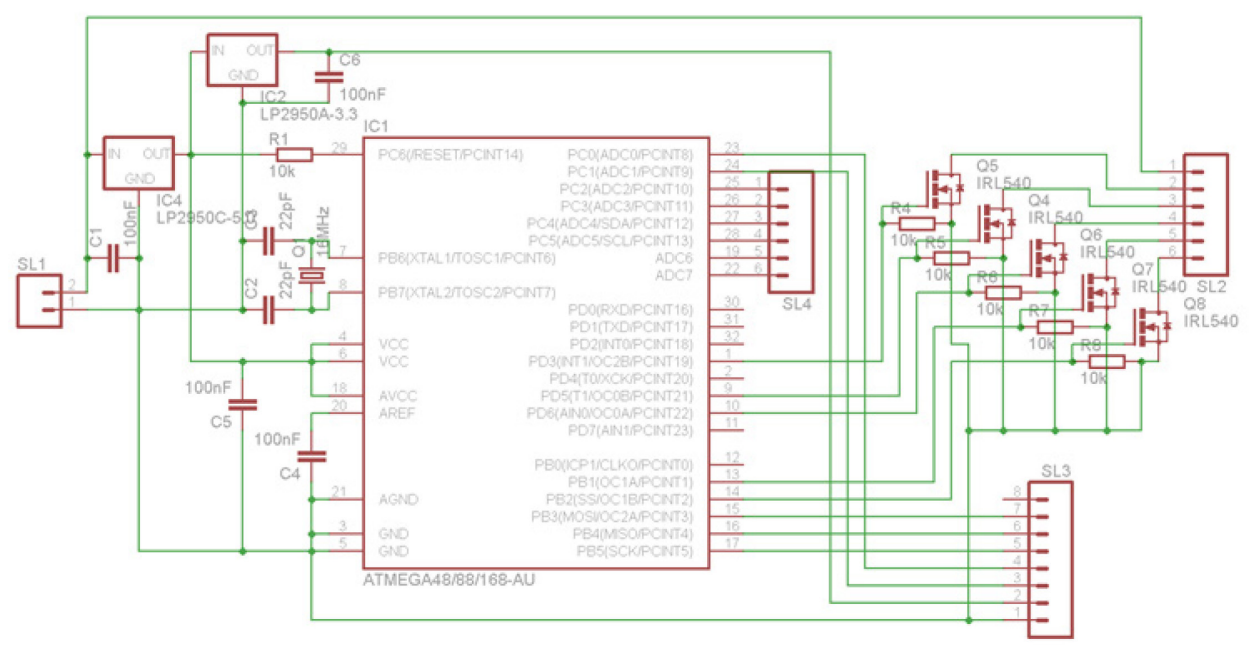

Figure 3. Circuit diagram of the controller 


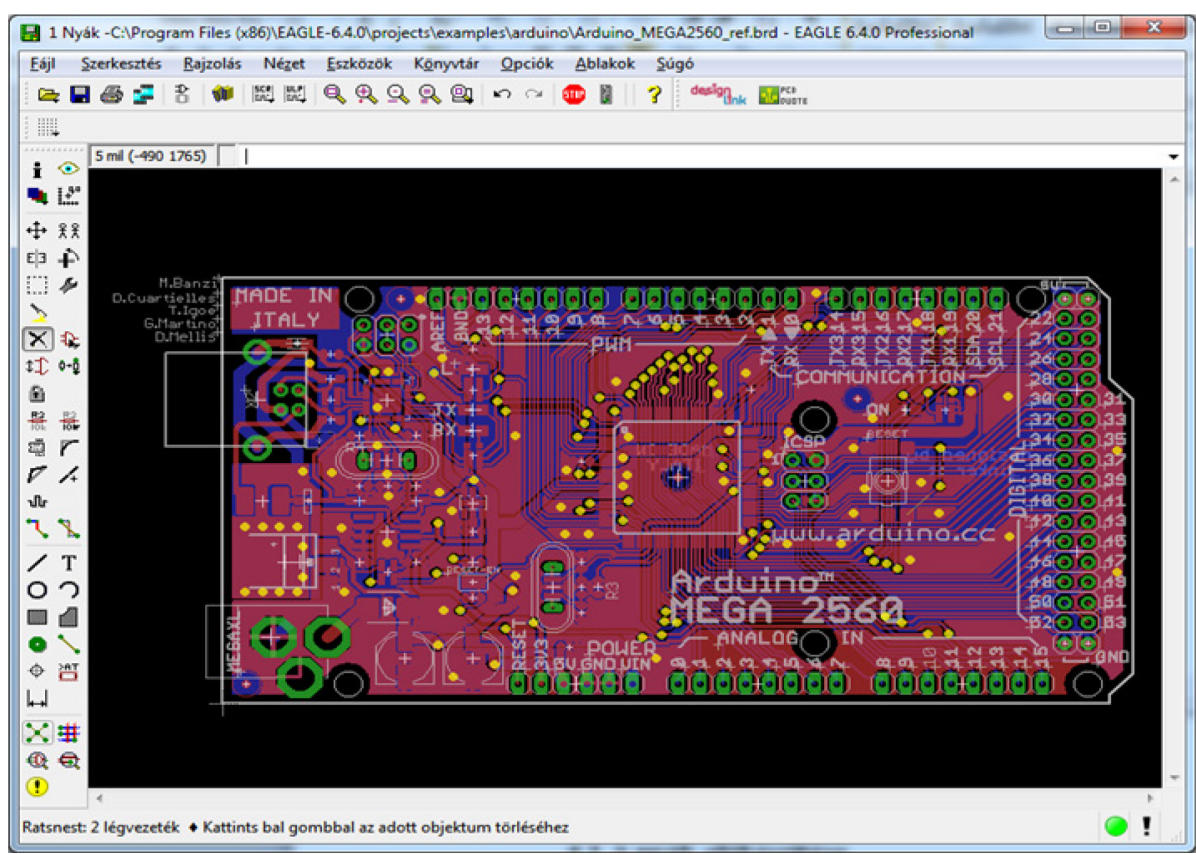

Figure 4. Printed-circuit using EAGLE

Once the chandelier is powered on it should illuminate with a set light intensity just as a regular chandelier, after the initial power up the light intensity and the lighting mod can be set with the remote controller. Thus in the initialization part in the program these variables have to be set to their default values and the input and output pins have to be set, since by default all pins are set to inputs. Furthermore the default state of the outputs needs to be set (high, low), for the inputs we can also set the pullup resistor which ensures the stability of the input if no signal is connected. The next task is to set the PWM generator with the appropriate registers, the module is configured for 8 bit precision, fast PWM generation [9], [11], [12].

Finally the watchdog timer needs to be started, the task of which is to restart the microcontroller if the execution of the program stops or the microcontroller freezes. This is a counter which works on an internal independent clock source, if this counter goes beyond a certain value (overflows) it can set a bit or restart the microcontroller. Once the initialization is complete the main program is run periodically. The objective of this code is to set the light intensity of the LEDs, control the radio module, interpret the incoming packets and to coordinate the execution of the program.

The control of the colour LED strips can be simplified if the three base colours (red, green, blue) are not set individually but rather as HSL (Hue, Saturation, Lightness) values. This way only three variables are needed the colour, the intensity, and the brightness which is more user friendly than RGB colour mixing. The final step after writing the code for the microcontroller and assembling the circuit was the assembly and placement of the chandelier.

\section{LED CHANDELIER}

The designed and implemented LED chandelier became the jewel of the living quarters (Fig. 5). Once built the chandelier's functionality could not be fully utilized, since the unique electronics required a remote that could not be purchased commercially, this prompted the building of a remote controller. Once built, all the functionalities have been tested. The required light intensities were reached. The mood lighting could be set to illuminate the room with daylight intensity all the way to evening dimness. 


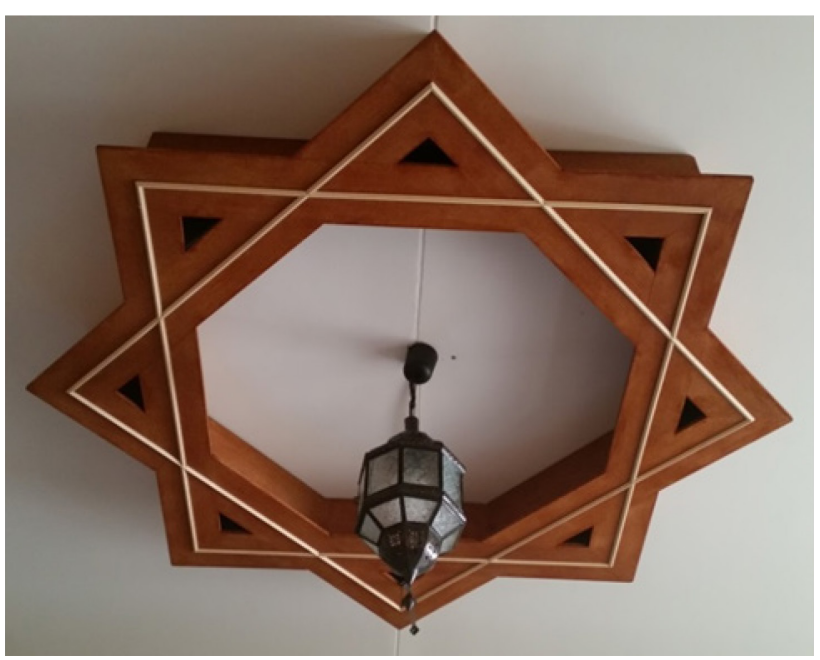

Figure 5. Completed chandelier

\section{CONCLUSION}

Light is life, the story of lighting will follow that of humanity, not much more than a century has passed since Thomas Edison and his lightbulb still lighting technology has advanced in leaps and bounds. The impressiveness of LED technology lies within the generation of light itself. The light emitting diodes compared to traditional light sources offer vibration free, clear light. The quality of LED lighting can be seen immediately as lighting expenses decrease once installed in a home.

\section{REFERENCES}

[1] D. McCrea, Using LED's in your own projects, Everything you need to know, Amazon Digital Services LLC, 2014, $29 \mathrm{p}$.

[2] L. Madarász, 30 éves a LED, Rádiótechnika évkönyve, 2003, pp. 96-113.

[3] A. Arató, Világítástechnikai kislexikon, Világítástechnikai Társaság, Budapest, 2001, pp. 72-73.

[4] B. Hanus, Napenergiával müködő világítódiódák, Cser Könyvkiadó és Ker. Kft., 2012, 100 p.

[5] S. Cangeloso, LED Lighting: A Primer to Lighting the Future, Maker Press, 2012, 70 p.

[6] Cs. Ugróczky, K. Csányi, A jövő fényforrásai a jelenben, VillanyÁsz, 2 (2007), pp. 24-25.

[7] O. Kückmann, High-power LED arrays: special requirements on packaging technology, LightEmitting Diodes: Research, Manufacturing, and Applications X, 6134 (2006), 8 p.

[8] K. C. Lahue, Interior Lighting, Ortho Books, 1991, 112 p.

[9] ATmega48A/PA/88A/PA/168A/PA/328/P Datasheet, 2015

[10]M. A. Mazidi, S. Naimi, S. N. Mazidi, AVR Microcontroller and Embedded Systems: Using Assembly and C, Prentice Hall Press, 2010, 792 p.

[11]T. Grace, Programming and Interfacing ATMEL's AVRs, Cengage Learning PTR, 2015, 272 p.

[12]H. W. Huang, The Atmel AVR Microcontroller: MEGA and XMEGA in Assembly and C, Delmar Cengage Learning; 1 edition 2013, 848 p. 